\title{
Accurate Calibration of Microscope Magnification Standard
}

\author{
J.A. Kramar, G.W. Witzgall, V.P. Scheuerman, M.T. Postek \\ National Institute of Standards and Technology, Gaithersburg, MD 20899-8212
}

A necessary condition for quantitative measurements with any instrument is accurate calibration. Accurate length measurement requires that the calibration be traceable to the unit of length as defined by the International System of Units (SI). Commercial artifacts are available for microscope calibration purposes, some providing SI traceability through comparison to primary standards that have been measured at a National Metrology Institute (NMI), such as the National Institute of Standards and Technology (NIST). These are secondary standards, calibrated by comparison to the primary standard with an increased uncertainty of the measurement. Opportunities exist for decreasing the uncertainties in the primary standards, especially at the smaller distances, by using new instrumentation being developed at NIST.

NIST is developing a unique instrument that can be used for establishing the SI traceability of calibration artifacts at the millimeter to sub-micrometer scale. The Molecular Measuring Machine $\left(\mathrm{M}^{3}\right)$ is designed to measure two-dimensional (2D) feature placement over a $50 \mathrm{~mm}$ by $50 \mathrm{~mm}$ area (see Fig. 1). [1] The probe is a scanning probe microscope (SPM), which achieves sub-nanometer resolution. The position of the probe is measured by a heterodyne Michelson interferometer system, also having sub-nanometer resolution. SI traceability is provided via the known frequency of the interferometer laser. The instrument is contained in a high vacuum environment, thus eliminating any uncertainty in the interferometry arising from the refractive index of air. $\mathrm{M}^{3}$ also features a temperature control system that can maintain better than $0.01 \mathrm{C}$ temperature stability. Beyond resolution and stability, measurement uncertainty depends on the full characterization of the measuring instrument, including axis alignments and cross-couplings. This characterization is still in process for $\mathrm{M}^{3}$, but some meaningful measurements are nevertheless possible. Special test measurements can be made with the currently achievable measurement uncertainty. The total measurement uncertainty will also include components that depend on the quality of the artifact and the nature of the particular measurement.

As part of the $\mathrm{M}^{3}$ development process, we have undertaken the task of measuring pitch features on a commercial calibration artifact, the Geller MRS-4 [2]. We have focused initially on the finest resolution target on the artifact, the 2D $500 \mathrm{~nm}$ pitch pattern, which is made up of concentric boxes ranging from $2 \mu \mathrm{m}$ to $40 \mu \mathrm{m}$ in $1 \mu \mathrm{m}$ increments. In order to position the SPM probe over the $50 \mathrm{~mm}$ range with sub-nanometer resolution, $\mathrm{M}^{3}$ uses stacked coarse and fine motion stages. The range of the fine motion stages is $10 \mu \mathrm{m}$. We have therefore in general imaged nine, overlapping, $5.5 \mu \mathrm{m}$ by $5 \mu \mathrm{m}$ images across the central section of the $2 \mathrm{D}$ grid using the fine motion stages, and from these have extracted the average pitch. The coarse motion stage was moved between each image. Each of the images was taken under closed-loop, interferometer-based servo control. Note that the interferometer system directly measures the combined coarse and fine motion in 2D, as illustrated in Fig. 1, so no stitching is necessary to measure from image to image. The image

* This work was performed by U.S. government employees, and is not subject to U.S. copyright. 
sequences were taken in both axes. An image of the center of the pattern from a $\mathrm{Y}$ axis measurement is shown in the Fig 2.

To extract the pitch, the line locations must first be defined based on the image data. We have done this using an iterative, weighted averaging method. The pitch measurements from line to line were then statistically analyzed. Corrections to the extracted pitch were made based on known axis crosscouplings, particularly the Z-to-Y and Z-to-X coupling. The net result is an extracted pitch of 501 $\mathrm{nm}$ in both axes, with an estimated standard uncertainty of $5 \mathrm{~nm}$. We find the pitch of this particular specimen to be within our measurement uncertainty of the nominal value.

In the future, we plan to measure additional selected small pitch features on this calibration artifact. We are also quite interested in measuring some of the larger features. This would allow corroboration of the M3 measurements with other accurate metrology instruments at NIST.

\section{References}

[1] John Kramar, et al., Proceedings of the SPIE Conference on Metrology, Inspection, and Process Control for Microlithography XIII, SPIE Vol. 3677, (1999) 1017

[2] Certain commercial equipment, instruments, or materials are identified in this abstract in order to specify the experimental procedure adequately. Such identification is not intended to imply recommendation or endorsement by NIST, nor is it intended to imply that the materials or equipment identified are necessarily the best available for the purpose.

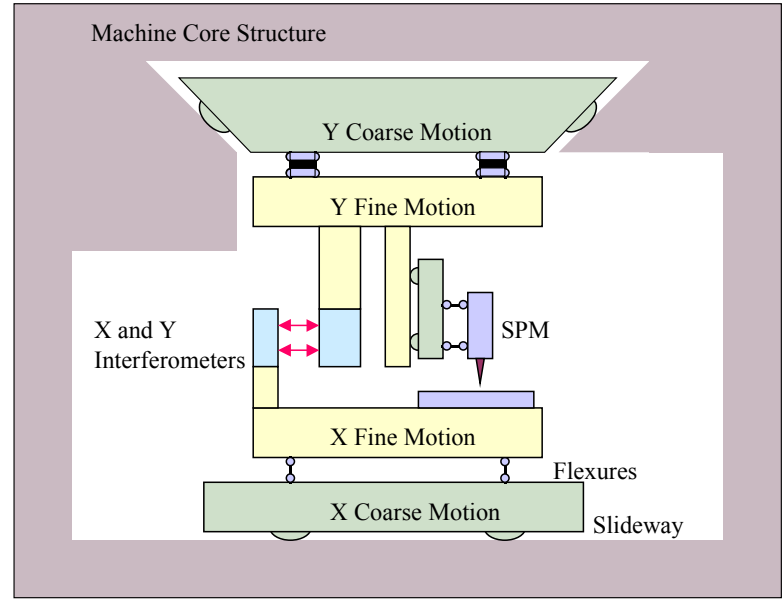

FIG. 1. Schematic block diagram of $\mathrm{M}^{3}$.

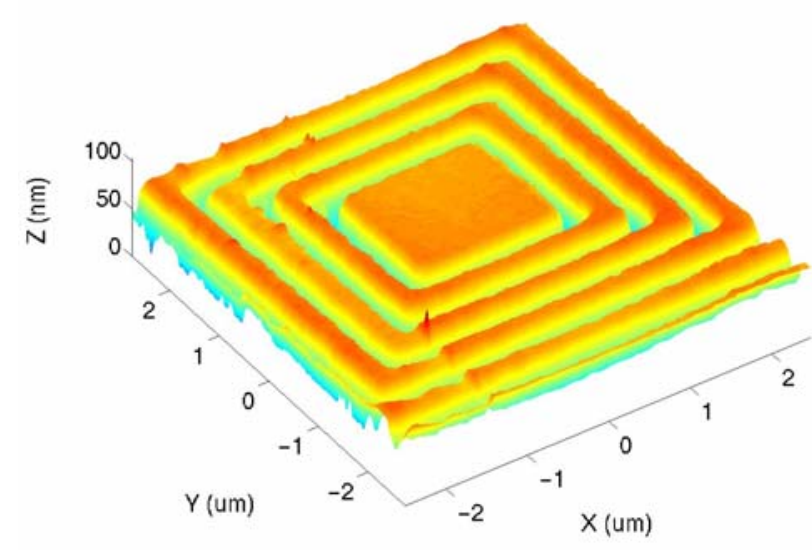

FIG. 2. $\mathrm{M}^{3}$ micrograph of central portion of $500 \mathrm{~nm}$ pitch 2D box in box pattern. 